\title{
The Intelligent Control System for infocommunication networks
}

\author{
Lubov Berkman $^{1}$, Oleg Barabash ${ }^{2}$, Olga Tkachenko $^{3}$, AndriMusienko ${ }^{4}$, Oleksand Laptiev $^{5}$, Ivanna Salanda $^{6}$ \\ ${ }^{1}$ Doctor of Technical Sciences, Professor, Vice-rector for scientific and pedagogical work, State University of \\ Telecommunications, Ukraine,090289@ukr.net \\ ${ }^{2}$ Doctor of Technical Sciences, Professor, Head of Department of Mathematics, State University of Telecommunications, \\ Ukraine, bar64@ukr.net \\ ${ }^{3}$ Doctor of Technical Sciences, Associate Professor, Department of Telecommunication Systems and Networks,State University of \\ Telecommunications, Ukraine, okar@ukr.net \\ ${ }^{4}$ Doctor of Technical Sciences, Associate Professor, Department of Mathematics, State University of Telecommunications, \\ Ukraine, mysienkoandrey@gmail.com \\ ${ }^{5}$ Philosophy Doctor, Senior Researcher, Associate Professor, Department of Information and Cybersecurity Systems, State \\ University of Telecommunications, Ukraine, alaptev64@ukr.net \\ ${ }^{6}$ Philosophy Doctor, Associate Professor, Department of Theory and Methods of Labor Education and Technology,Ternopil \\ Regional Council Taras Shevchenko Regional Humanitarian-Pedagogical Academy of Kremenets, \\ Ukraine, salanda.ivanna@gmail.com
}

\begin{abstract}
The conceptual model of the intellectual network, which is proposed to be used in the synthesis of the control information transmission network in the intelligent systems of management of the infocommunication networks, is investigated. It is shown that the following features must be taken into account during the synthesis of Intelligent Control Systems (ICS): the processing speed at the upper levels of the conceptual model decreases with the growth of "intelligence", which in turn falls as we move down the transportation level of the proposed model. The principles of construction and architecture of the ICS are presented, taking into account the current readings of measuring and information means. A model for servicing a claim in ICS is proposed, which uses the use of controllability determinants and centralized logic. Dynamic distribution of information flows has been shown to be one of the effective ways to increase the use of network resources in an emergency. In order to ensure dynamic flow distribution in the control information transmission network, it is proposed to use a dynamic flow distribution management system, which is a subsystem of ICS. Dynamic routing has been shown to be effective only with average channel usage.
\end{abstract}

Key words: Network, intellectualization, control system, synthesis, model, requirement, impact, distribution, flow.

\section{INTRODUCTION}

The management of modern information and communication networks should be independent of the services that are provided to the consumer. For the interaction of distributed control components in a single system, as well as for the implementation of management functions, a network is needed to transmit control information.
The development of the concept of intelligent control systems (ICS) raises a number of fundamental issues. The first of them is associated with the form of presentation of information for the organization of management mechanisms. At the same time, the most important aspect is the analysis of the possibilities and features of the use of various information technologies for processing information in intelligent control tasks.

For parametric and structural adjustment of control algorithms, modification of the program to achieve control goals, and if necessary, their correction, taking into account the current readings of measuring and information tools, the principles of building the ICS are proposed.

The need for effective identification of emergencies, providing significant capabilities for analysis and recognition of the deviation of the parameters of control objects from the norm, necessitate the use of a hierarchical organization of ISU. The formation of a strategy of appropriate behavior, planning a sequence of actions, the synthesis of executive laws that satisfy the specified quality indicators, is associated with the distribution of intellectual functions between the levels of the network. This ensures the selection of a unique set of proprietary models for the presentation of information, their processing mechanisms, means of information support and expectations of management objects.

The main selection criterion should be the necessary quality of the control system functioning under conditions of uncertainty with the random nature of external disturbances, which include unforeseen changes in goals, own operational characteristics, environmental parameters, etc.

\subsection{Literature analysis and problem statement}

In recent years, developers are increasingly having to solve the problems of designing object management systems of a rather complex nature. Objects for which there is a need to develop a new concept for constructing control systems is 
commonly called "large systems." Unfortunately, there is no clear definition of large systems that would suit all professionals. Quite often in the literature you can find only those characteristic features that do not allow adhering to traditional methods developing in the theory of identification. This is confirmed by the works ([1], [2]).

In [3], the basic methods of the theory of optimal control are considered: dynamic programming, the Pontryahin minimum principle, numerical optimization methods. These methods are the basis for building control systems. However, the work does not show the possibility of using the considered methods for constructing intelligent control systems.

In [4], a comparative characteristic of information structures in group decision problems and their influence on the flexibility of group optimization are presented. This is an important issue at the stage of synthesis of complex technical systems. Moreover, the results of the study for emergency situations are not covered.

In the literature [5], the term "intelligent system" was formed, which emphasizes the main difference between such systems - the possibility of systematic information processing. However, the issues of autonomy, adaptability and reliability of control systems in the presence of various kinds of uncertainties are not fully disclosed.

The publication [6] presents the features of the synthesis of a TMN second-level communication network management system with a combined control principle, and the control principles for perturbation and deviation are considered in detail. Moreover, the results of the study for the case of intelligent systems are not covered.

It was shown in [7] that network control systems are spatially distributed systems for which communication between controllers and actuators is supported by a common communication network. However, the issues of choosing the main indicators of the quality of the control channel are not fully disclosed.

Publication [8] is devoted to the study of the architecture of an intelligent network, the development of methods for calculating the basic parameters of a network, the relationships between them and vector network synthesis. At the same time, it is not shown which methods of vector synthesis are appropriate to use when optimizing the intelligent network.

In [9], a new technological approach for the architecture of the Internet of things (IoT) networks was demonstrated. It involves a multi-tier implementation using cloud servers, optimized data streaming and loading. This will significantly reduce the transmission time, costs and memory of the main cloud server.

In [10], an optimal reception algorithm was proposed using a coherent reception method, which is advisable to use in control channels of infocommunication networks. Moreover, the research results for the case of intellectual networks are not covered.

$\operatorname{In}[11]$, the directions of the development of infocommunications as a developing extension of telecommunications with the processing of information and content of the management function were investigated.
Information and communications technology is considered an extended synonym for information technology to emphasize the integration of telecommunications. However, the management of infocommunication networks is not fully disclosed.

The article [12] explores the main points of the theory of automatic control. The stages of its formation as a science are given. The terminology and conceptual ideas of the construction of closed systems, as well as the theory of perturbations, stability and synthesis of self-tuning systems as part of the general theory of automatic control are considered. However, the synthesis and control of intelligent systems are not considered in the work.

The article [13] explores the technology of building 5G mobile networks, which should provide the conditions for the creation of ultra-dense networks for high-quality services. A method for optimizing signal parameters by a uniform criterion is developed. This method can be used to build an intelligent infocommunication network management system.

Based on the analysis of literary sources, the following conclusions can be drawn. In the study, analysis and synthesis of information and communication networks, the apparatus of complex systems is effective. The creation of systems a priori oriented to work in conditions of incompleteness or fuzziness of the initial information, the uncertainty of external disturbances and the operating environment requires the use of non-traditional approaches to control using artificial intelligence methods and technologies. Such systems, called intelligent control systems, actually create a new class for which the principles of construction, analysis and synthesis methods should take into account all the characteristic features of heterogeneous telecommunication networks of the future.

\subsection{Aim of the article}

The aim of the work is to develop the concept of building intelligent control systems to ensure the effective, flexible and reliable functioning of infocommunication networks.

To achieve this goal it is necessary to solve the following tasks:

- formalization of the synthesis problem of the transmission network of control information according to the conceptual model of an intelligent network;

- building and researching the requirements service model in an intelligent management system;

- application of dynamic distribution of information flows in an intelligent control system.

\section{THE MAIN SECTION}

\subsection{Intelligent network conceptual model}

The problem of managing infocommunication networks is one of the most important in the practice of operating networks. The requirements for a modern control system for an infocommunication network are significantly different from previously known ones. The user of the digital future should be able to not only receive a variety of high-quality services, but also create the services he needs [1]. To provide 
this opportunity, it is necessary to synthesize an intelligent control system (ICS). The main property of the ICS is the fact that the control information transmission network is built according to the conceptual model of an intelligent network (Figure 1).

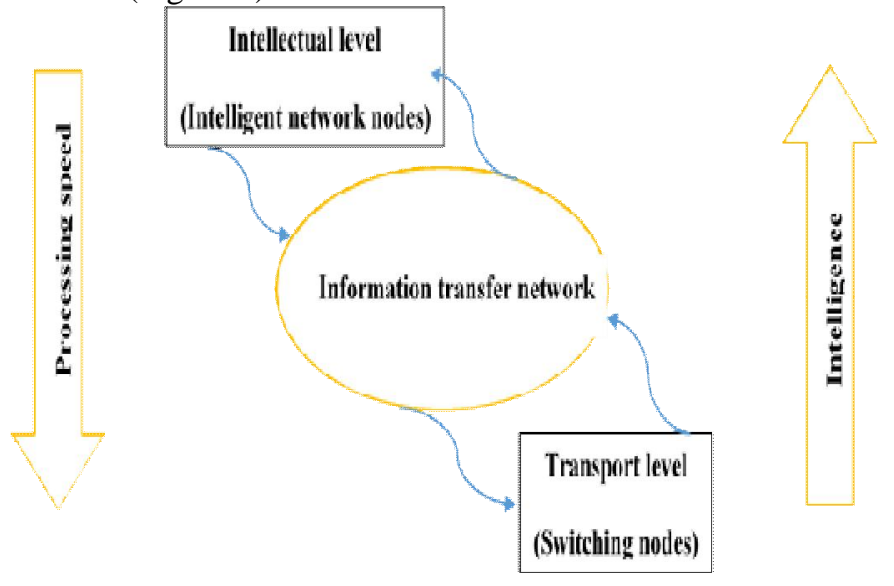

Figure 1: Intelligent network conceptual model

Intelligent systems are characterized by two significant features. Processing speed decreases with the growth of "intelligence", which, in turn, decreases as you move down the transport level of the proposed model. This causes those problems that require an optimal solution when using the concept of an intelligent network [2].

Thus, taking into account modern requirements, it is proposed to build infocommunication network management systems using the methods and technologies of intelligent networks. The main property of intelligent control systems is the fact that the control information transmission network is built according to the conceptual model of an intelligent network. With this approach to the synthesis of the control information transmission network, the processing speed at the upper levels decreases with the growth of "intelligence", which, in turn, decreases as it moves down the transport level of the proposed model.

\subsection{The requirements service model in the intelligent control system}

The requirements service model in the ICS, shown in Figure 2 , implies the introduction of control actions determinants these are additional software and / or hardware tools that facilitate the further expansion of functions and changes to any system.

The requirements service model in the ICS includes three main components:

- $\quad$ basic requirements processing unit;

- a control action determinant that recognizes applications forwarded to the ICS and temporarily suspends the requirements service process for the period of information exchange with the ICS logic control unit;

- ICS logical control unit containing hardware and software designed to create additional control actions and transmit information that controls standard requirements processing processes.

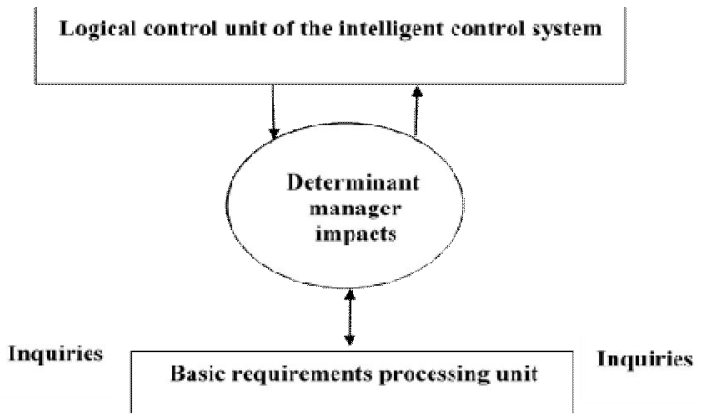

Figure2. The requirements service model in the ICS

This separation of requirements servicing functions in an intelligent system has several advantages. To perform the functions of a determinant of the control action, only a small correction of the existing switching systems is necessary, which is implemented by the corresponding software. The central logical control unit allows you to speed up and simplify the process of entering new and correcting existing control actions, which affects the efficiency of the entire network. It is proposed to consider the ICS conceptual model as a structure of three planes (levels) (Figure 3). The model separates control commands and aspects related to the network, which allows you to describe the capabilities of an intelligent network regardless of the core network over which the intelligent add-on is created. The first level is control commands. The second level - functional - describes the capabilities of the network that are necessary for the implementation of services. The network is considered as a whole, requirements processing models and structural blocks independent of control commands are given. The third level physical - describes the nodes of the network, the functional elements contained in them and the interaction protocols. At this level, physical elements are determined, ways of mapping functional elements to physical ones, and ways to implement network elements of an intelligent network are described.Blocks independent of control commands provide standard reusable functions. The basic process of processing requirements is carried out by a specialized unit that interacts with other units through points of initialization and completion.

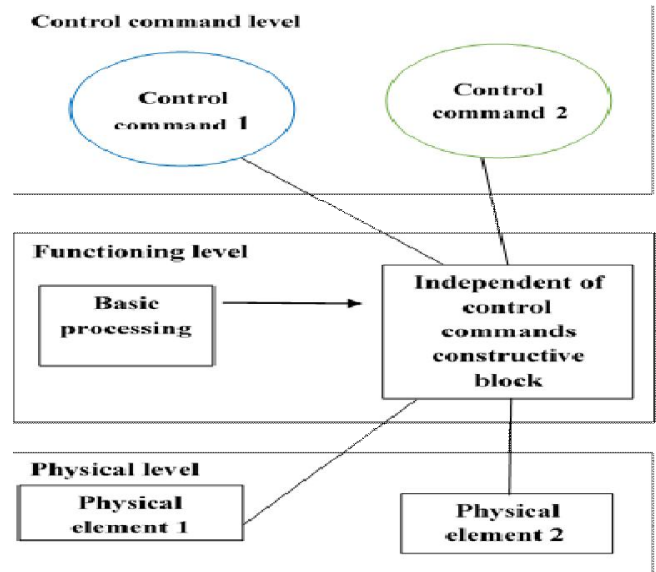

Figure 3: Conceptual model ICS

The main requirements for the structure of an intelligent network are: 
- network functions are performed in nodes of an intelligent network;

- a node can perform one or more functions;

- the execution of a common network function cannot be carried out jointly by several nodes;

- two different nodes can perform the same network functions;

- nodes must have standard interfaces;

- the distribution of network functions across nodes and standard interfaces should not be network dependent.

Thus, a model for servicing requirements in the IMS is proposed, which implies the introduction of determinants of the control action and centralized logic, which allows to speed up and simplify the process of introducing new and correcting existing control actions. This makes it possible to increase the efficiency of the entire network.

\subsection{Dynamic distribution of information flows in the ICS}

One of the effective ways to increase the use of network resources in emergency conditions is the dynamic distribution of information flows [14].

To ensure the dynamic distribution of flows in the control information transmission network, it is proposed to use a system of dynamic control of the distribution of flows, which is a subsystem of the ICS.

Packet transfer with dynamic control of packet flows is provided not only along the direct path, but also along any of the available workarounds depending on the network situation [13].

The probability of occupying the resources of this path is due to the probability of occupying the memory resources of the network nodes through which this path passes, and the probability of occupying the virtual channels, which is characterized by the utilization factor $\rho, 0 \leq \rho \leq 1$. With a uniform load on the entire network, the probability of the presence of a busy main, but free one workaround, that is, the probability of choosing a workaround for packet transmission can be calculated as follows: $\mathrm{P} \approx \rho(1-\rho)$. Probability of choosing $\mathrm{N}-1$ workarounds: $\mathrm{P} \approx \rho(1-\rho \mathrm{N}-1)$. In Figure4 shows graphs of the dependence of the probability of choosing a workaround $\mathrm{P}$ on the channel utilization factor $\rho$ in the presence of two, three and four acceptable directions (paths) of information transfer. As can be seen from Pic. 4, the probability $\mathrm{P}$ of choosing one of the $\mathrm{N}-1$ detours is small, both for small values of $\rho$ (the load is small, so there is no need to use not a direct, but a detour), and for large values of $\rho$. Since the workaround is usually a longer path, selecting it for transmission leads to an increase in the overall coefficient use of channels.

Assume that the utilization rate increases uniformly across the network by $\alpha$, then

$P \approx \rho(1+\alpha)(1-[\rho(1+\alpha)] N-1)$.

It should be noted that this dependence is satisfied for $\rho<1 /(1+\alpha)$

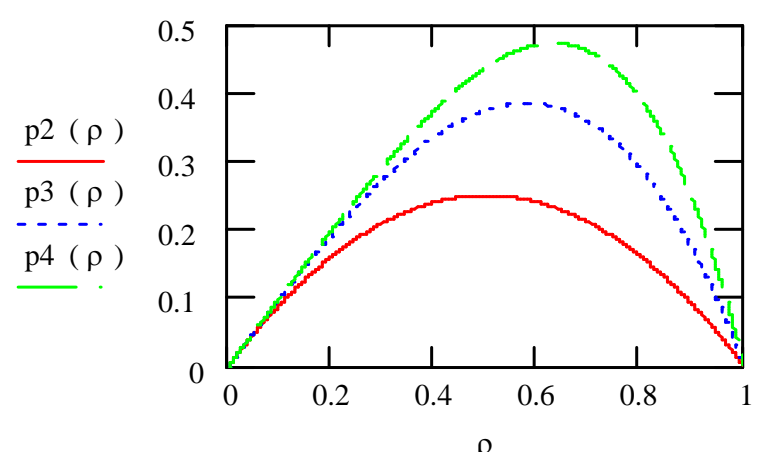

Figure 4: Graphs of the dependence of the probability P of choosing a workaround on the utilization rate of channels $\rho$ in the presence of two, three, and four acceptable directions

(paths) of packet transmission

In Figure. 5 shows plots of the dependence of $\mathrm{P}$ on $\rho$ when using workarounds, given that $\alpha=20 \%$.

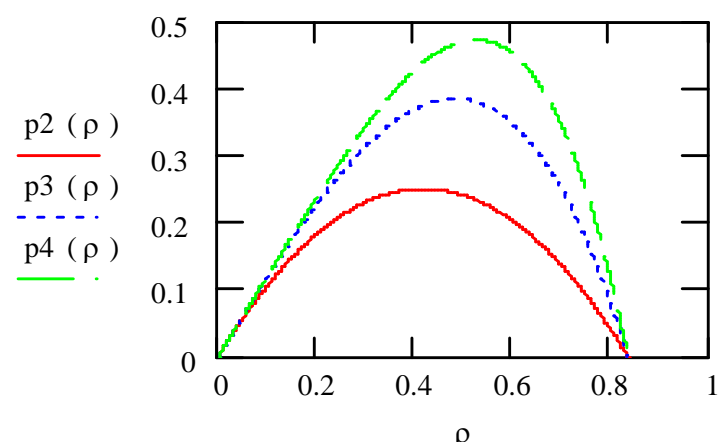

Figure 5: Graphs of the dependence of $P$ on $\rho$ when using workarounds for $\alpha=20 \%$

Thus, one of the effective ways to increase the use of network resources in emergency conditions is the dynamic distribution of information flows. To ensure the dynamic distribution of flows in the control information transmission network, it is proposed to use a system of dynamic control of the distribution of flows, which is a subsystem of the ICS. To confirm the effectiveness of dynamic routing, studies were conducted of the dependence of the probability of choosing bypass information transmission routes on the channel utilization factor. It is shown that dynamic routing is effective only with the average use of channels.

\section{RESULT AND DISCUSSION}

\subsection{Research results of the average delay time in the control information transmission network}

The average delay time of a message in the network, including the waiting time for transmission over channels, with a static distribution of packet transmission paths:

$\tau_{S T}=H \times L /(1-\rho)$,

where $\mathrm{H}$ is the average number of transit sections in the selected transmission path.

The calculation by formula (2) implies the following restrictions: Poisson message flows in the network, an 
exponential distribution of message lengths with an average number of packets $\mathrm{L}$, and a normalized unit time of transmission of a packet over a channel.

With dynamic distribution of packet streams, two options are possible: either packets of one message are waiting for the channel to be released in the originally selected path for the message (virtual channel network), or transmitted along each of the roundabouts (datagram mode).

For the first case:

$\tau_{D 1}=H \times L /[(1-\rho(1+\alpha)]$,

(3)

where $(1+\alpha)$ takes into account the fact that part of the load during dynamic control of the distribution of packet flows will be transmitted along longer paths.

In the second case, for the condition that there is no waiting for transmission over the channels, the delay time of a message in the network consists of the time required to transmit L packets from the first (initial) node, and the transmission time of each packet from $\mathrm{H}(1+\alpha)-1$ other (transit) nodes. Assuming that the time taken to transmit one packet is equal to one, the total time required to transmit $\mathrm{L}$ packets from the source node is equal to L units.

Since in the datagram mode all packets of one message are transmitted over the network in parallel in different ways, it can be assumed that the transmission time of all packets through the transit nodes of the network is numerically equal to the transmission time of one packet over the network. Thus, in the datagram mode, the transmission time of packets through the transit nodes takes $\mathrm{H}(1+\alpha)-1$ units of time. Thus,

$\tau_{D 2}=L+H(1+\alpha)-1$.

Given (2) for the main path and (4) for workarounds, we find the total average delay time of messages in the network with the dynamic distribution of packet flows:

$\tau_{D}=(1-P) H /(1-\rho)+P[L-1+H(1+\alpha)]$.

(5)

In Figure 6 shows graphs of the dependence of the average network delay time on the channel utilization in the case of static (constant routes) and dynamic (dynamic routing) packet distributions at $\alpha=20 \%$ and two $(\mathrm{N}=2)$ and four ( $=4$ ) valid paths packet transfer.

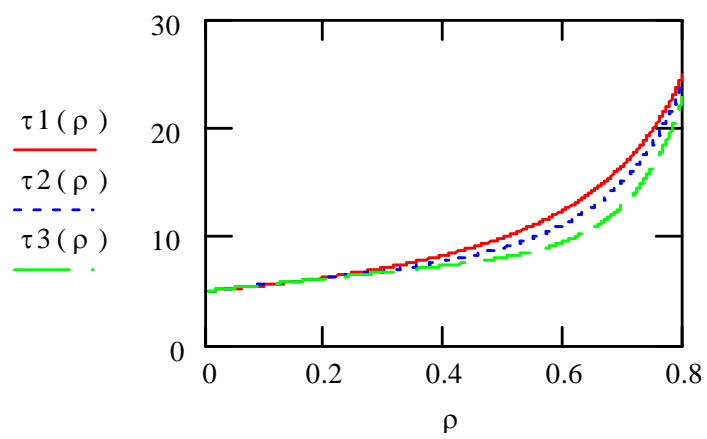

Figure 6: Graphs of the average network delay time versus channel utilization coefficient: curve $\tau 1(\rho)$ - for the case of static packet distribution; curve $\tau 2(\rho)$ - for the case of dynamic distribution of packets at $\alpha=20 \%$ and $\mathrm{N}=2$; curve $\tau 3(\rho)$ - for the case of dynamic distribution of packets at $\alpha$ $=20 \%$ and $\mathrm{N}=4$
Graphs analysis in Figure 6 shows that dynamic routing is only effective with average channel utilization.

However, it is worth noting that this comparison of the dynamic and static distributions of packet flows is carried out to evenly distribute the load on an absolutely reliable network. In case of load distortions and / or any damage on the network, the dynamic distribution of packet flows leads to a significantly greater effect.

Thus, the dynamic control of the distribution of information flows is most effective in emergency situations on the network. Moreover, in conditions of significant network congestion, the use of only one dynamic control of the distribution of information flows can not only not improve, but even worsen the quality of message transmission (message transmission delay). Therefore, dynamic control of the distribution of information flows must be applied in combination with other methods, and, first of all, with the method of controlling the amount of information flow entering the network.

\subsection{Discussion of research results on the principles of building an intelligent control system for infocommunication networks}

Given the trends in the rapid development of telecommunication networks, requirements are set for a modern control system that differ significantly from previously known ones $[5,6]$. The user of the digital future should be able to not only receive a variety of high-quality services, but also create the services he needs [1]. The proposed ICS allows you to not only effectively manage, but also create a wide variety of services. This is achieved due to the fact that the control information transmission network is built on the basis of an intelligent network from blocks independent of services $([2,13])$.

The concept of an intelligent network allows creating scenarios of a wide variety of services for each user with a limited amount of databases, which is especially important for the digital economy.

The advantage of intelligent control systems in practice is that the proposed model for servicing requirements in the ICS implies the introduction of determinants of the control action and centralized logic, which makes it possible to speed up and simplify the process of introducing new and correcting existing control actions, which affects the efficiency of the entire network.

The main advantage of the proposed approach is that the introduction of determinants of the control action facilitates the further expansion of functions and the introduction of changes to any system.

Compared to the combined control principle [6], the proposed approach provides choice of unique together their own models of knowledge representation, mechanisms for their processing and updating, information support tools and expectations of facilities management.

In addition, unlike the techniques proposed in [8], it becomes possible to increase the use of network resources in emergency conditions using dynamic distribution of information flows. 
At the same time, it should be noted that the possibilities of applying the proposed approach to the construction of a control system are limited by the need for a priori knowledge of the network structure.

Dynamic control of the distribution of information flows is most effective in emergency situations on the network. However, in conditions of significant network congestion, the use of only one dynamic control of the distribution of information flows may not only not improve, but even worsen the quality of message transmission. This position requires the integrated use of the dynamic method with other methods of controlling the distribution of information flows.

Further development of the proposed principle of dynamic control of the distribution of information flows consists in the need to apply it in combination with other methods, and, first of all, with the method of controlling the amount of information flow entering the network.

\section{CONCLUSION}

Given the requirements of the present and especially the fact that the user of the digital future should be able not only to receive various high-quality services, but also to create the services he needs, it is proposed to build infocomunication network management systems using the methods and technologies of intelligent networks. The main property of intelligent control systems is the fact that the control information transmission network is built according to the conceptual model of an intelligent network. With this approach to the synthesis of the control information transmission network, the processing speed at the upper levels decreases with the growth of "intelligence", which, in turn, decreases as it moves down the transport level of the proposed model.

A model for servicing requirements in the ICS is proposed, which implies the introduction of determinants of the control action and centralized logic, which allows to speed up and simplify the process of introducing new and correcting existing control actions, which affects the efficiency of the entire network.

One of the effective ways to increase the use of network resources in emergency conditions is the dynamic distribution of information flows. To ensure the dynamic distribution of flows in the transmission network of control information, it is proposed to use a system of dynamic control of the distribution of flows, which is a subsystem of the ICS. It has been determined that dynamic routing is only effective with average channel utilization. In case of load distortions and / or any damage on the network, the dynamic distribution of packet flows leads to a significantly greater effect.

\section{REFERENCES}

1. Sezer S. et al. Are we ready for SDN Implementation challenges for software-defined networks // IEEE Commun. Mag. Jul. 2013. Vol. 51. Issue 7. P. 36-43.

https://doi.org/10.1109/MCOM.2013.6553676
2. Gupta R. A., Chow M.-Y. Networked control system: overview and research trends // IEEE Transactions on Industrial Electronics. 2010. Vol. 57. Issue 7. P. 2527-2535.

3. Kirk D. E. Optimal control theory: An introduction. Mineola, New York: Dover, 2004. 452 p.

4. Mahajan A., Martins N. C., Rotkowitz M. C., Yuksel S. Information structures in optimal decentralized control // Proceedings of the IEEE Conference on Decision and Control. 2012. P. 1291-1306.

https://doi.org/10.1109/CDC.2012.6425819

5. Kulin M., Fortuna C., De Poorter E., Deschrijver D., Moerman I. Data-Driven Design of Intelligent Wireless Networks: An Overview and Tutorial // Sensors. 2016. Issue 16(6). P. 790-796.

6. Aaron Don M. Africa, Lourdes Racielle Bulda, Emmanuel Del Rosario, Matthew Zandrick Marasigan and Isabel Navarro, Radio Wave Propagation: Simulation of Free Space Propagation Path Loss. International Journal of Emerging Trends in Engineering Research( IJETER ) // 2020. Vol. 8. P.281-287.

https://doi.org/10.30534/ijeter/2020/07822020

7. Hespanha J. P., Naghshtabrizi P., Xu Y. A Survey of Recent Results in Networked Control Systems // Proceedings of the IEEE. 2007. Issue 95 (1). P. 138-162.

8. Kibria M. G. et al. Big Data Analytics, Machine Learning, and Artificial Intelligence in Next-Generation Wireless Networks // 2018. Vol. 6. P. 1284-1291.

https://doi.org/10.1109/ACCESS.2018.2837692

9. Memos V. A. Efficient Multimedia Transmission over Scalable IoT Architecture // International Journal of Computer Network and Information Security (IJCNIS). 2018. Vol. 10. Issue 6. P. 27-39.

https://doi.org/10.5815/ijcnis.2018.06.03

10. V Venkata Narayana, Sk Hasane Ahammad, B. Vinay Chandu, G.Rupesh, G.Abishek Naidu and G.Pavan Gopal, Estimation of Quality and Intelligibility of a Speech Signal with varying forms of Additive Noise. International Journal of Emerging Trends in Engineering Research(IJETER) // 2019.Vol.7. P.430-433.

https://doi.org/10.30534/ijeter/2019/057112019

11. Sallai Gy. Defining Infocommunications and Related Terms // Acta Polytechnica Hungarica (ISSN 1785-8860). 2012. Vol. 9. Issue 6. P. 5-15.

12. Varun K L Srivastava, N. Chandra Sekhar Reddy and Dr. Anubha Shrivastava, An efficient Software Source Code Metrics for Implementing for Software quality analysis International Journal of Emerging Trends in Engineering Research (IJETER) // 2019.Vol. 7 No. 9 P.216-222.

https://doi.org/10.30534/ijeter/2019/01792019

13. Laptiev Oleksandr, Shuklin German, Savchenko Vitalii, Barabash Oleg, Musienko Andrii and Haidur Halyna, The Method of Hidden Transmitters Detection based on the Differential Transformation Model. International Journal of Advanced Trends in Computer Science and Engineering (IJATCSE). Vol. 8, No 6, (November - December 2019). P. $2840-2846$.

https://doi.org/10.30534/ijatcse/2019/26862019 